sicherer wollen sie uneingeschränkt anbieten können, die Ärzte lehnen das ab. Dabei ist folgender Kompromiss denkbar: Versicherer bieten nur dort Listenmodelle an, wo keine Vertragsmodelle möglich sind. Bei Vertragsmodellen gilt das Tariffestsetzungsverfahren nach KVG, falls sich die Partner nicht einigen können. Während des Festsetzungsverfahrens werden die AVM unverändert weitergeführt. Dadurch soll wie bei der konventionellen Grundversicherung verhindert werden, dass die betroffenen Versicherten Nachteile in
Kauf nehmen müssen, wenn sich die Tarifpartner nicht einigen können.

Integrierte Versorgungsmodelle können nicht von einem Partner des Gesundheitswesens alleine vorgeschlagen und entwickelt werden. Intelligente Kompromisse können nur im Dialog der Partner entwickelt und entscheidungsreif vorbereitet werden. Deshalb braucht es einen «runden Tisch» mit interessierten Teilnehmern: Patienten, Politik (Bund und Kantone), Versicherer, Ärztenetzwerke und Ärzteschaft.

\title{
De nouveaux visages à la rédaction du BMS
}

\section{Bruno Kesseli, Chefredaktor}

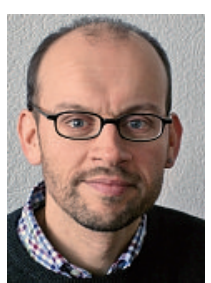

Thomas Heuer

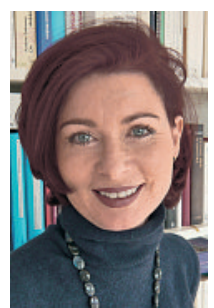

Annette Eichholtz

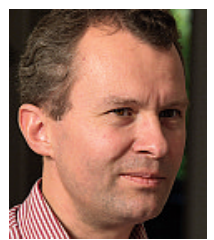

Christoph Rehmann-Sutter

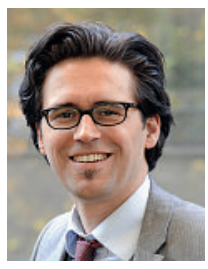

Rouven Porz
Deux changements ont eu lieu à la rédaction du Bulletin des médecins suisses le 1er juin. Voici une brève information à ce sujet. Annette Eichholtz et Rouven Porz occupent désormais les postes de Thomas Heuer et Christoph Rehmann-Sutter.

\section{Thomas Heuer :}

\section{en route vers de nouveaux horizons}

Il n'est pas facile d'imaginer la rédaction du BMS sans lui. Pendant plus de 11 ans, Thomas Heuer a veillé, en qualité de Managing Editor du BMS, à ce que les procédures se déroulent sans accros, de la remise des manuscrits à la production des articles prêts à imprimer et à ce que les lectrices et les lecteurs disposent chaque semaine d'une revue répondant aux standards de publication des Editions médicales suisses. Lorsque des problèmes sont survenus, il a, pendant environ six mois, assumé le commandement. Toujours sur le pont, il a mené le BMS tout au long de la période d'avril à octobre 2005 où aucun rédacteur en chef n'était en charge de cette mission.

Thomas Heuer s'est désormais lancé dans une activité indépendante et vogue vers de nouveaux rivages. Nous remercions très cordialement notre aimable collègue pour le travail fourni et lui souhaitons «bon vent» pour l'avenir.

Vous trouverez une rétrospectives de ses 11 années et demie au BMS, par Thomas Heuer, dans la rubrique «Et encore» de ce numéro (dernière page du Bulletin).

\section{Annette Eichholtz: du «brainstorm» au BMS}

Même si nous perdons du savoir-faire avec le départ de Thomas Heuer, nous n'aurons pas à craindre son absence. En effet, avec Annette Eichholtz M.A., c'est une collègue hautement qualifiée, disposant d'une riche expérience de la publication, qui a pris la relève comme Managing Editor. Après des études dans les sciences de l'éducation, la philologie allemande et la philosophie, Annette Eichholtz a travaillé pendant 12 ans comme lectrice pour un éditeur. Par la suite, elle a dirigé la revue neuro-oncologique brainstorm de l'association allemande «Hirntumorhilfe» pendant huit ans, en tant que rédactrice en chef. Nous souhaitons une cordiale bienvenue à notre nouvelle collègue et lui présentons tous nos vœux de début d'activité réussi.

Annette Eichholtz se présentera aux lecteurs du BMS dans la rubrique «Et encore» du numéro 25 du 17 juin.

\section{Christoph Rehmann-Sutter: nomination à Lübeck}

C'est un événement en soi réjouissant qui est la cause du regrettable départ de Christoph Rehmann-Sutter de l'unité d'éthique de la rédaction du BMS. Sa nomination largement relayée par les médias comme professeur de théorie de la médecine et d'éthique biomédicale à l'université de Lübeck (Allemagne) a exigé de lui une réorientation et une concentration des efforts qui l'ont incité à renoncer non seulement à son mandat de président et de membre de la Commission nationale d'éthique mais également à son poste de rédacteur au BMS. Avec le départ de Christoph Rehmann-Sutter, la rédaction perd un membre extrêmement apprécié et compétent qui, en dépit de ses nombreuses tâches, s'est toujours distingué par sa disponibilité et a beaucoup aidé les auteurs à améliorer leurs travaux grâce à des commentaires de relecture critiques mais toujours constructifs. Christoph Rehmann-Sutter ne sera pas totalement perdu pour la rédaction et les lecteurs du BMS dans la mesure où, à l'avenir encore, il publiera quelques articles dans le BMS.

Une interview complète de Christoph Rehmann-Sutter paraîtra dans l'un des prochains numéros du BMS.

\section{Rouven Porz: une garantie de continuité}

En ce qui concerne le départ de Christoph RehmannSutter, nous sommes également à la fois tristes et joyeux. C'est en effet avec plaisir que nous accueillons son successeur Rouven Porz. Tout comme Christoph Rehmann-Sutter qui était son directeur de thèse de doctorat à Bâle, Rouven Porz est aussi bien biologiste que philosophe, une combinaison particulièrement précieuse pour la rédaction du BMS au vu de la complexité toujours plus importante des questions d'éthiques biomédicale. De plus, en tant que responsable du service d'éthique de l'Hôpital de l'Île à Berne, Rouven Porz est très proche de la réalité clinique à laquelle les médecins sont confrontés quotidiennement. Ceci pourrait l'aider également dans son travail pour le Bulletin des médecins suisses. La rédaction lui souhaite une cordiale bienvenue.

Rouven Porz se présentera lui aussi aux lecteurs du BMS dans l'un des prochains numéros, à la rubrique «Et encore». 